\title{
Acessibilidade no parto e nascimento a pessoas com deficiência motora, visual ou auditiva: estrutura de estabelecimentos do SUS vinculados à Rede Cegonha
}

\author{
Accessibility to people with motor, visual, or hearing disabilities \\ during delivery and childbirth: the structure of SUS facilities linked \\ to Rede Cegonha
}

Erika Barbara Abreu Fonseca Thomaz (https://orcid.org/0000-0003-4156-4067) ${ }^{1}$

Elisa Miranda Costa (https://orcid.org/0000-0001-5364-0384) ${ }^{1}$

Yara Naya Lopes de Andrade Goiabeira (https://orcid.org/0000-0002-8982-9549) ${ }^{1}$

Thiago Augusto Hernandes Rocha (https://orcid.org/0000-0002-6262-3276) ${ }^{2}$

Núbia Cristina Silva Rocha (https://orcid.org/0000-0002-0809-2152) ${ }^{3}$

Maria Cristina de Oliveira Marques (https://orcid.org/0000-0001-6550-3360) ${ }^{4}$

Rejane Christine de Sousa Queiroz (https://orcid.org/0000-0003-4019-2011) ${ }^{1}$

${ }^{1}$ Departamento de Saúde Pública, Universidade Federal do Maranhão. R. Barão de Itapary 155 Centro. São Luís MA Brasil.

erika.barbara@ufma.br

${ }^{2}$ Organização Pan-

Americana de Saúde.

Brasília DF Brasil.

${ }^{3}$ Methods, Analytics and

Technology for Health

(M.A.T.H. Consortium)

${ }^{4}$ Ministério da Saúde, Brasil.

Brasília DF Brasil.

\begin{abstract}
There are no nationwide studies characterizing accessibility for people with disabilities during delivery. This study aimed to describe the physical structure of hospital units regarding accessibility for pregnant and puerperae with motor $(M D)$, visual (VD), or hearing (HD) disabilities in Brazil. This is an ecological, descriptive study conducted in all 606 health facilities linked to the "Rede Cegonha" where deliveries occurred, according to 2015 databases. We performed the descriptive and geospatial analysis and considered the presence of motor accessibility when the establishment had a handrail or elevator ramp, wheelchair-sized doors, and accessible bathroom with bars. We assumed visual accessibility when there was tactile signage on the floor (Braille system or embossed figures) and hearing accessibility when there was signage by texts, pictures, signs, posters, or symbols in the environments. In Brazil, only 26 (4.3\%) of the facilities had accessibility for people with MD, 20 (3.3\%) for people with VD, and none for HD. Motor accessibility was worse in the North and Northeast of Brazil, and hearing accessibility in the North region. Despite advances in the implementation of the "Rede Cegonha" in Brazil, the facilities' structure is not adapted for women with $M D, V D$, or $H D$.
\end{abstract}

Key words People with disabilities, Maternal health services, Service structure
Resumo Não há estudos, de abrangência nacional, caracterizando a acessibilidade para pessoas com deficiência no momento do parto. O objetivo do estudo foi descrever a estrutura física de estabelecimentos hospitalares quanto à acessibilidade para gestantes e puérperas com deficiência motora (DM), visual (DV) ou auditiva (DA) no Brasil. Estudo ecológico, descritivo, realizado em todos os 606 estabelecimentos vinculados à Rede Cegonha $(R C)$, que realizaram partos em 2015. Foram feitas análises descritivas e de distribuição espacial. Considerou-se acessibilidade motora quando o estabelecimento tivesse rampa com corrimão ou elevador, portas com dimensões para cadeira de rodas e banheiro acessivel com barras; acessibilidade visual quando houvesse sinalização tátil (sistema Braille ou figuras em relevo); e acessibilidade auditiva quando houvesse sinalização por textos, figuras, placas, cartazes ou simbolos nos ambientes. No Brasil, apenas 26 (4,3\%) estabelecimentos tinham acessibilidade para pessoas com DM, 20 (3,3\%) para pessoas com DA e nenhum para pessoas com DV. A acessibilidade motora foi pior no Norte e Nordeste e a auditiva, no Norte. Apesar dos avanços decorrentes da implantação da $R C$ no Brasil, a estrutura dos estabelecimentos hospitalares não está adaptada para pessoas com DM, DV ou DA.

Palavras-chave Pessoas com deficiência, Serviços de saúde materna, Estrutura dos serviços 


\section{Introdução}

No último censo de 2010, vinte e quatro por cento da população brasileira referiu ter algum tipo de deficiência motora (DM), visual (DV) ou auditiva (DA). Mais de 13 milhões $(7,1 \%)$ tinham algum grau de deficiência motora, dos quais $734.421(0,4 \%)$ eram completamente incapacitados de deambular. Deficiência visual foi reportada por $35.774 .392(18,8 \%)$ brasileiros e, destes, $506.377(0,3 \%)$ eram completamente cegos. Algum grau de deficiência auditiva foi referido por 9.717.318 (5,1\%) brasileiros, dos quais 344.206 $(0,2 \%)$ não ouviam de modo algum. Estas deficiências são mais expressivas em mulheres do que em homens, especialmente motora e visual ${ }^{1}$.

Mulheres com deficiências experimentam múltiplas iniquidades sociais, econômicas e de saúde, com maior exposição à pobreza, desemprego e baixa escolaridade ${ }^{2-8}$. Mulheres grávidas com deficiências são mais suscetíveis a problemas na gestação e parto ${ }^{9-11}$. Ainda assim, estudos internacionais apontam que estas mulheres enfrentam barreiras e desafios relacionados ao acesso e à qualidade dos serviços de saúde reprodutiva e de prevenção ao câncer ${ }^{4,6,7,12-18}$.

Visando à diminuição das múltiplas vulnerabilidades vivenciadas pelas pessoas com deficiência, em 2002 foi instituída a Política Nacional de Saúde da Pessoa Portadora de Deficiência ${ }^{19}$, porém foi alterada em 2010, pela Política Nacional de Saúde da Pessoa com Deficiência ${ }^{20}$, já que o entendimento do conceito relativo à deficiência vem evoluindo em todo o mundo. No Brasil, a Rede de Cuidados à Pessoa com Deficiência, instituída a partir da Portaria No 793, de 24 de Abril de $2012^{21}$, enfatiza a necessidade de modificar a estrutura dos estabelecimentos de saúde no âmbito do Sistema Único de Saúde (SUS), garantindo o acesso, o aprimoramento da estrutura física e a qualificação profissional de toda a Rede de Cuidados, desde a atenção primária até a alta complexidade, incluindo financiamento destinado a este fim, visando a atender aos usuários dos serviços com deficiência.

Martins et al. ${ }^{22}$ verificaram que a estrutura física de UBS, em João Pessoa, Nordeste do Brasil, não estava adaptada para as pessoas com deficiências, divergindo dos princípios de equidade e universalidade do SUS. Em uma análise de 240 UBS em 41 municípios brasileiros com mais de 100 mil habitantes, Siqueira et al. ${ }^{23}$, verificaram que cerca de $60 \%$ das unidades eram inadequadas para o acesso de pessoas com deficiências. A presença de degraus, a falta de corrimãos, rampas, banheiros adaptados para os cadeirantes e salas de espera inadequadas às necessidades foram os problemas mais identificados.

Para a atenção ao parto, não foram identificados trabalhos que tenham avaliado a acessibilidade, em maternidades brasileiras, para gestantes/ puérperas com DM, DV ou DA. Todavia, há indícios de que a adequação da estrutura nas maternidades impacte na redução da mortalidade materna e infantil e nos desfechos perinatais ad$\operatorname{versos}^{24,25}$.

$\mathrm{Na}$ perspectiva de maior inclusão social e construção de uma cidadania plena e efetiva das pessoas com deficiência, no ano de 2015, o Brasil instituiu a Lei Brasileira de Inclusão da Pessoa com Deficiência, também conhecida como Estatuto da Pessoa com Deficiência ${ }^{26}$. Essa Lei garante acesso à saúde a essa parcela da população ${ }^{26}$. Entretanto, apesar da escassez de evidências, é possível supor que ainda persistem grandes desafios para garantir às mulheres com deficiências o acesso universal e equitativo aos serviços de saúde durante o parto e o nascimento em estabelecimentos da rede SUS.

Tendo por referência esse desafio e dada a escassez de trabalhos que se debruçassem sobre esse tema, o objetivo deste trabalho é descrever a estrutura física de estabelecimentos hospitalares vinculados à Rede Cegonha (RC) quanto a aspectos da acessibilidade para gestantes e puérperas com deficiência motora, visual ou auditiva no Brasil.

\section{Método}

\section{Delineamento do estudo, unidade de análise}

Trata-se de um estudo ecológico, descritivo, cuja a coleta dos dados foi realizada em um único momento no tempo, entre de dezembro de 2016 e setembro de 2017. As unidades de análise foram os estabelecimentos hospitalares, públicos ou conveniados ao SUS, vinculados à RC.

\section{Amostra e local do estudo}

Foram incluídos todos os hospitais que, em 2015, conforme dados do Sistema de Informações de Nascidos Vivos (SINASC), preenchiam os seguintes critérios: i) Realização de 500 ou mais partos e localização em região de saúde com plano de ação da RC, independentemente da liberação de recursos $(\mathrm{N}=581)$; e ii) Realização de menos de 500 partos, localizada em região de saúde com plano de ação da RC e com liberação de recursos da $\mathrm{RC}(\mathrm{N}=25)$. Dessa forma, a população do estudo foi composta por 606 estabelecimentos, sendo 86 no Norte, 175 no Nordeste, 223 no Sudeste, 81 no 
Sul e 41 no Centro-Oeste. Estes estabelecimentos foram identificados pelo linkage entre o SINASC e o Cadastro Nacional de Estabelecimentos de Saúde (CNES).

As regiões brasileiras têm indicadores socioeconômicos e de saúde contrastantes. Em 2017, a região Norte tinha taxa de analfabetismo de $8,8 \%{ }^{27}$ e Produto Interno Bruto (PIB) de R\$ 337.231 bilhões $^{28}$. Possuía a maior razão de mortalidade materna (RMM) do Brasil, com 76,0 óbitos por 100 mil nascidos vivos ${ }^{29}$, seguido da região Nordeste. O Nordeste tinha PIB de R\$ 898.082 bilhões $^{28}$, taxa de analfabetismo de $14,3 \%{ }^{27}$ e a segunda maior RMM, com 75,3 por 100 mil nascidos vivo ${ }^{29}$. O Sudeste possui as mais altas concentrações demográficas, urbanísticas e melhores indicadores econômicos. Tem o maior PIB do país (R\$ 3.332 trilhões $)^{28}$, a menor taxa de analfabetismo $(3,8 \%)^{27}$ e a RMM, em 2015 , era de 54,3 por 100 mil nascidos vivos ${ }^{29}$. O Sul tinha PIB de R\$ 1.066 trilhão $^{28}$ e RMM de 43,7 por 100 mil nascidos vivos $^{29}$. O Centro-Oeste tinha PIB de R\$ 632.889 bilhões $^{28}$, taxa de analfabetismo de $6,8 \%{ }^{27}$ e RMM de 65,9 por 100 mil nascidos vivos ${ }^{29}$.

\section{Coleta de dados}

Os dados foram coletados por meio de observação in loco utilizando um Roteiro de Observação, especialmente desenvolvido para este estudo. A coleta foi feita em formulário digital, utilizando o software REDCap® 2017, Vanderbuilt University. Este roteiro foi fundamentado na Política Nacional de Humanização ${ }^{30}$, Política Nacional da $\mathrm{RC}^{31}$, nas normas das Boas Práticas de Atenção ao Parto e Nascimento ${ }^{32}$, Diretrizes de Atenção à Gestante: a operação cesariana ${ }^{33}$ e Diretrizes de Atenção à Gestante: parto normal ${ }^{34}$. O objetivo da observação in loco foi avaliar processos de cuidado e as condições de infraestrutura, planta física, equipamentos, materiais e insumos do estabelecimento hospitalar.

A observação do estabelecimento foi previamente agendada com gestores do estado, do município e do próprio estabelecimento. Estes foram convidados a participar do processo ou enviar um representante no dia da coleta dos dados, de modo a percorrerem o estabelecimento hospitalar, junto com o avaliador, durante o registro das informações.

\section{Variáveis do estudo}

As variáveis relacionadas à acessibilidade de gestantes e puérperas com deficiência motora, visual ou auditiva foram extraídas do instrumento e constam do Quadro 1. Dados relativos a variáveis contextuais dos hospitais foram: região geopolítica, Unidade Federativa (UF), município, além de dados das coordenadas geográficas dos hospitais.

Para fins de análise, foram criadas variáveis de acessibilidade motora, visual ou auditiva para cada hospital e para cada ambiente do estabelecimento hospitalar, conforme descrito no Quadro 1. Considerou-se presença de Acessibilidade Motora no hospital quando, durante a observação in loco, foi verificada acessibilidade em todos os ambientes analisados (Quadro 1). O mesmo se aplica para a Acessibilidade Visual e Acessibilidade Auditiva. Para a análise de acessibilidade visual e auditiva, o alojamento conjunto não foi considerado por ausência dessa informação nas bases de dados que utilizamos para o presente estudo.

\section{Análise estatística}

A análise descritiva dos dados foi realizada calculando-se frequências absolutas e relativas para cada variável dentro do estabelecimento hospitalar. A partir daí, observou-se a proporção de maternidades em cada UF com os itens adequados. Essas informações foram utilizadas para produzir análises de distribuição espacial por UF.

Para caracterizar a distribuição espacial dos estabelecimentos hospitalares de acordo com a acessibilidade de pessoas com DM, DV ou DA foram confeccionados mapas com a distribuição espacial dos pontos em seus respectivos municípios a partir das coordenadas geográficas obtidas por meio do endereço dos estabelecimentos hospitalares. As análises descritivas foram realizadas no software Stata ${ }^{\circledR}$, versão 14.0 (StataCorp., College Station, Estados Unidos). Os mapas por pontos foram confeccionados no software ArcGIS , versão10.5 (Esri Inc., 2017, Estados Unidos) e os mapas temáticos no software Q-GIS ${ }^{\circledast}$, versão $3 \cdot 10.1^{35}$. Para visualizar a área de alcance dos estabelecimentos classificados com acessibilidade a pessoas com DM, DV ou DA, juntos, foi confeccionado mapa com linhas de distância entre esses estabelecimentos em relação aos todos os demais estabelecimentos existentes no Brasil. Foram obtidos raios de alcance a partir da adoção de três parâmetros de distancias $(30 \mathrm{~km}, 50 \mathrm{~km}$ e $100 \mathrm{~km})$ a partir dos estabelecimentos com acessibilidade a pessoas com DM, DV ou DA.

\section{Considerações éticas}

A pesquisa foi aprovada pelo Comitê de Ética em Pesquisa com Seres Humanos da Universidade Federal do Maranhão e da Escola Nacional de 
Quadro 1. Descrição das variáveis do estudo. Brasil, 2017.

\begin{tabular}{|c|c|c|}
\hline & Ambiente do hospital & Critérios para classificação das variáveis \\
\hline \multirow[t]{4}{*}{ 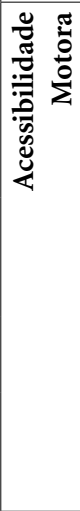 } & Recepção & $\begin{array}{l}\text { Considerou-se presença de acessibilidade motora na recepção quando o } \\
\text { ambiente estivesse adequado para as variáveis: } \\
\checkmark \text { Rampa(s) com corrimão ou elevador; } \\
\checkmark \text { Porta(s) com dimensões para o tamanho de cadeira de rodas; } \\
\checkmark \text { Banheiro acessível com barras. }\end{array}$ \\
\hline & Sala de admissão & $\begin{array}{l}\text { Considerou-se presença de acessibilidade motora na sala de admissão quando } \\
\text { o ambiente estivesse adequado para a variável: } \\
\checkmark \text { Porta(s) com dimensões para o tamanho de cadeira de rodas. }\end{array}$ \\
\hline & Unidade obstétrica $^{1}$ & \multirow{2}{*}{$\begin{array}{l}\text { Considerou-se presença de acessibilidade motora para cada um desses } \\
\text { ambientes (unidade obstétrica e alojamento conjunto) quando o ambiente } \\
\text { estivesse adequado para as variáveis: } \\
\checkmark \text { Porta(s) com dimensões para o tamanho de cadeira de rodas; } \\
\checkmark \text { Banheiro acessível com barras. }\end{array}$} \\
\hline & Alojamento conjunto & \\
\hline \multirow{3}{*}{ 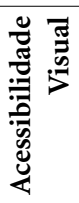 } & Recepção & \multirow{3}{*}{$\begin{array}{l}\text { Considerou-se presença de acessibilidade visual para cada um desses } \\
\text { ambientes (recepção, sala de admissão e unidade obstétrica) quando o } \\
\text { ambiente estivesse adequado para a variável: } \\
\checkmark \text { Existência da sinalização tátil no chão (sistema Braille ou figuras em } \\
\text { relevo). }\end{array}$} \\
\hline & Sala de admissão & \\
\hline & Unidade obstétrica $^{1}$ & \\
\hline \multirow{3}{*}{ 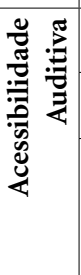 } & Recepção & \multirow{3}{*}{$\begin{array}{l}\text { Considerou-se presença de acessibilidade auditiva para cada um desses } \\
\text { ambientes (recepção, sala de admissão e unidade obstétrica) quando o } \\
\text { ambiente estivesse adequado para as variáveis: } \\
\checkmark \text { Existência de sinalização através de textos ou figuras ou símbolos dispostos } \\
\text { em local destacado; } \\
\checkmark \text { Existência de informação, placas informativas (placas, cartazes } \\
\text { informativos no interior do ambiente). }\end{array}$} \\
\hline & Sala de admissão & \\
\hline & Unidade obstétrica $^{1}$ & \\
\hline
\end{tabular}

${ }^{1}$ Unidade obstétrica: salas coletivas de pré-parto (com ou sem separação entre os leitos) e/ou quarto PPP (pré-parto, parto e pósparto).

Saúde Pública Sérgio Arouca, em 14 de dezembro de 2016. Todos participantes assinaram Termo de Consentimento Livre e Esclarecido. A pesquisa foi financiada com recursos do Fundo Nacional de Saúde/Ministério da Saúde. Este artigo compõe parte da Pesquisa avaliativa da gestão e atenção ao parto e nascimento na rede SUS do Brasil.

\section{Resultados}

A estrutura dos estabelecimentos hospitalares/ maternidades vinculados à RC no Brasil não está adaptada para pessoas com deficiência motora (Tabela 1, Figura 1), visual (Tabela 2, Figura 1) ou auditiva (Tabela 3, Figura 1).

Apenas 26 estabelecimentos (4,3\%) apresentaram os quatro ambientes (recepção, sala de admissão, unidade obstétrica e alojamento conjunto) com as características mínimas de acessibilidade motora aferidas nesse estudo, conforme descrito no Quadro 1.

Ao considerar o indicador síntese de acessibilidade motora, os estabelecimentos do Centro -Oeste (\%) e Sudeste (\%) tiveram um desempe- nho melhor que as demais regiões - Sul, embora todas tenham proporções de ambientes acessíveis inferiores a $10 \%$. Os estados de Sergipe $(22,2 \%)$, Mato Grosso do Sul (18,2\%), Acre (16,7\%) e Mato Grosso (16,7\%) foram os que apresentaram melhor desempenho. A recepção - rampa(s) com corrimão ou elevador, portas com dimensões para o tamanho de cadeira de rodas e banheiro acessível com barras - foi o ambiente com pior estrutura para a acessibilidade motora (16,5\%). A sala de admissão - portas com dimensões para o tamanho de cadeira de rodas - obteve os melhores resultados de adequação $(65,2 \%)$ (Tabela 1, Figura 1).

Nenhum dos 606 estabelecimentos avaliados no Brasil possuía sinalização tátil no chão (sistema Braille ou figuras em relevo) na recepção, sala de admissão e unidade obstétrica. Sinalização tátil na recepção foi verificada em três estabelecimentos do Nordeste $(1,7 \%)$, em quatro do Sudeste $(1,8 \%)$ e em um do Sul $(1,2 \%)$ e um do Centro-Oeste (2,4\%). Na sala de admissão, apenas dois estabelecimentos do Sudeste $(0,9 \%)$ tinham sinalização tátil. Na unidade obstétrica apenas três estabelecimentos no Nordeste $(1,7 \%)$ 
Tabela 1. Acessibilidade a pessoas com deficiência motora das maternidades vinculadas à Rede Cegonha. Brasil, 2017.

\begin{tabular}{|c|c|c|c|c|c|c|}
\hline Unidade Federativa & $\begin{array}{l}\text { No de Ma- } \\
\text { ternidades } \\
\text { Avaliadas }\end{array}$ & $\begin{array}{c}\text { Recepção } \\
\text { n (\%) }\end{array}$ & $\begin{array}{c}\text { Sala de } \\
\text { Admissão } \\
\text { n (\%) }\end{array}$ & $\begin{array}{c}\text { Unidade } \\
\text { Obstétrica } \\
\text { n (\%) }\end{array}$ & $\begin{array}{c}\text { ALCON } \\
\text { n }(\%)\end{array}$ & $\begin{array}{c}\text { Indicador de } \\
\text { Acessibili- } \\
\text { dade Motora } \\
\text { n (\%) }\end{array}$ \\
\hline Norte & 86 & $6(7,0)$ & $35(40,7)$ & $10(11,6)$ & $10(11,6)$ & $2(2,3)$ \\
\hline Rondônia & 7 & - & $5(71,4)$ & $1(14,3)$ & - & $0(0,0)$ \\
\hline Acre & 6 & $2(33,3)$ & $4(66,7)$ & $2(33,3)$ & $2(33,3)$ & $1(16,7)$ \\
\hline Amazonas & 11 & $1(9,1)$ & $6(54,5)$ & $2(18,2)$ & $1(9,11)$ & - \\
\hline Roraima & 1 & $0(0,0)$ & $1(100,0)$ & - & - & - \\
\hline Pará & 47 & $2(4,3)$ & $14(29,8)$ & $3(6,4)$ & $5(10,6)$ & $1(2,1)$ \\
\hline Amapá & 7 & $1(14,3)$ & $1(14,3)$ & $1(14,3)$ & $2(28,6)$ & - \\
\hline Tocantins & 7 & - & $4(57,1)$ & $1(14,3)$ & - & - \\
\hline Nordeste & 175 & $21(12,0)$ & $119(7)$ & $25(14,3)$ & $35(20,0)$ & $5(2,9)$ \\
\hline Maranhão & 14 & $3(21,4)$ & $9(64,3)$ & $2(14,3)$ & $2(14,3)$ & - \\
\hline Piauí & 8 & - & $1(12,5)$ & $1(12,5)$ & $2(25,0)$ & - \\
\hline Ceará & 44 & $3(6,8)$ & $27(61,4)$ & $9(20,4)$ & $7(15,9)$ & $1(2,3)$ \\
\hline Rio Grande do Norte & 11 & - & $5(45,5)$ & $1(9,1)$ & $5(45,5)$ & - \\
\hline Paraíba & 10 & $2(20.0)$ & $8(80,0)$ & - & $2(20,0)$ & - \\
\hline Pernambuco & 32 & $4(12,5)$ & $26(81,3)$ & $3(9,4)$ & $5(15,6)$ & - \\
\hline Alagoas & 16 & $2(12,5)$ & $7(43,7)$ & $2(12,5)$ & $1(6,3)$ & $1(6,3)$ \\
\hline Sergipe & 9 & $2(22,2)$ & $9(100,0)$ & $3(33,3)$ & $3(33,3)$ & $2(22,2)$ \\
\hline Bahia & 31 & $5(16,1)$ & $27(87,1)$ & $4(12,9)$ & $8(25,8)$ & $1(3,2)$ \\
\hline Sudeste & 223 & $47(21,1)$ & $158(70,9)$ & $79(35,4)$ & $85(38,1)$ & $12(5,4)$ \\
\hline Minas Gerais & 44 & $15(34,1)$ & $32(72,7)$ & $22(50,0)$ & $24(54,6)$ & $4(9,1)$ \\
\hline Espírito Santo & 4 & - & $1(25,0)$ & - & $2(50,0)$ & - \\
\hline Rio de Janeiro & 41 & - & $29(70,7)$ & - & $16(39,0)$ & - \\
\hline São Paulo & 134 & $32(23,9)$ & $96(71,6)$ & $57(42,5)$ & $43(32,1)$ & $8(6,0)$ \\
\hline Sul & 81 & $10(12,4)$ & $60(74,1)$ & $25(30,9)$ & $30(37,0)$ & $3(3,7)$ \\
\hline Paraná & 23 & $2(8,7)$ & $18(78,3)$ & $7(30,4)$ & $8(34,8)$ & - \\
\hline Santa Catarina & 34 & $4(11,8)$ & $24(70,6)$ & $10(29,4)$ & $16(47,1)$ & $1(2,9)$ \\
\hline Rio Grande do Sul & 24 & $4(16,7)$ & $18(7)$ & $8(33,3)$ & $6(25,0)$ & $2(8,3)$ \\
\hline Centro-Oeste & 41 & $16(39,0)$ & $23(56,1)$ & $12(29,3)$ & $9(21,9)$ & $4(9,8)$ \\
\hline Mato Grosso do Sul & 11 & $5(45,5)$ & $9(81,8)$ & $5(45,4)$ & $3(27,3)$ & $2(18,2)$ \\
\hline Mato Grosso & 6 & $1(16,7)$ & $5(83,3)$ & $4(66,7)$ & $1(16,7)$ & $1(16,7)$ \\
\hline Goiás & 12 & $4(33,3)$ & $3(25,0)$ & $1(8,3)$ & $2(16,7)$ & - \\
\hline Distrito Federal & 12 & $6(50,0)$ & $6(50,0)$ & $2(16,7)$ & $3(25,0)$ & $1(8,3)$ \\
\hline Brasil & 606 & $100(16,5)$ & $395(65,2)$ & $151(24,9)$ & $169(27,9)$ & $26(4,3)$ \\
\hline
\end{tabular}

e três no Sudeste $(1,4 \%)$ tinham estrutura que permitisse a acessibilidade a gestantes/puérperas com deficiência visual (Tabela 2, Figura 1).

Observou-se a existência de sinalização através de textos, figuras ou símbolos em placas, cartazes informativos no interior da recepção, sala de admissão e unidade obstétrica em apenas em $20(3,3 \%)$ estabelecimentos hospitalares vinculados à RC. Os estabelecimentos do Norte apresentaram o pior desempenho - nenhum dos estabelecimentos preencheu os critérios de aces- sibilidade auditiva, de acordo com o indicador síntese (Tabela 3, Figura 1).

No mapa com raio de distância das maternidades com acessibilidade visual, motora ou auditiva, observou-se que, devido à inexistência de maternidades adequadas para os três indicadores de acessibilidade, dois estabelecimentos com acessibilidade motora e auditiva, que estão localizados nos estados da Bahia e Minas Gerais, alcançaram populações residentes dentro do próprio estado localizados nas distâncias de 

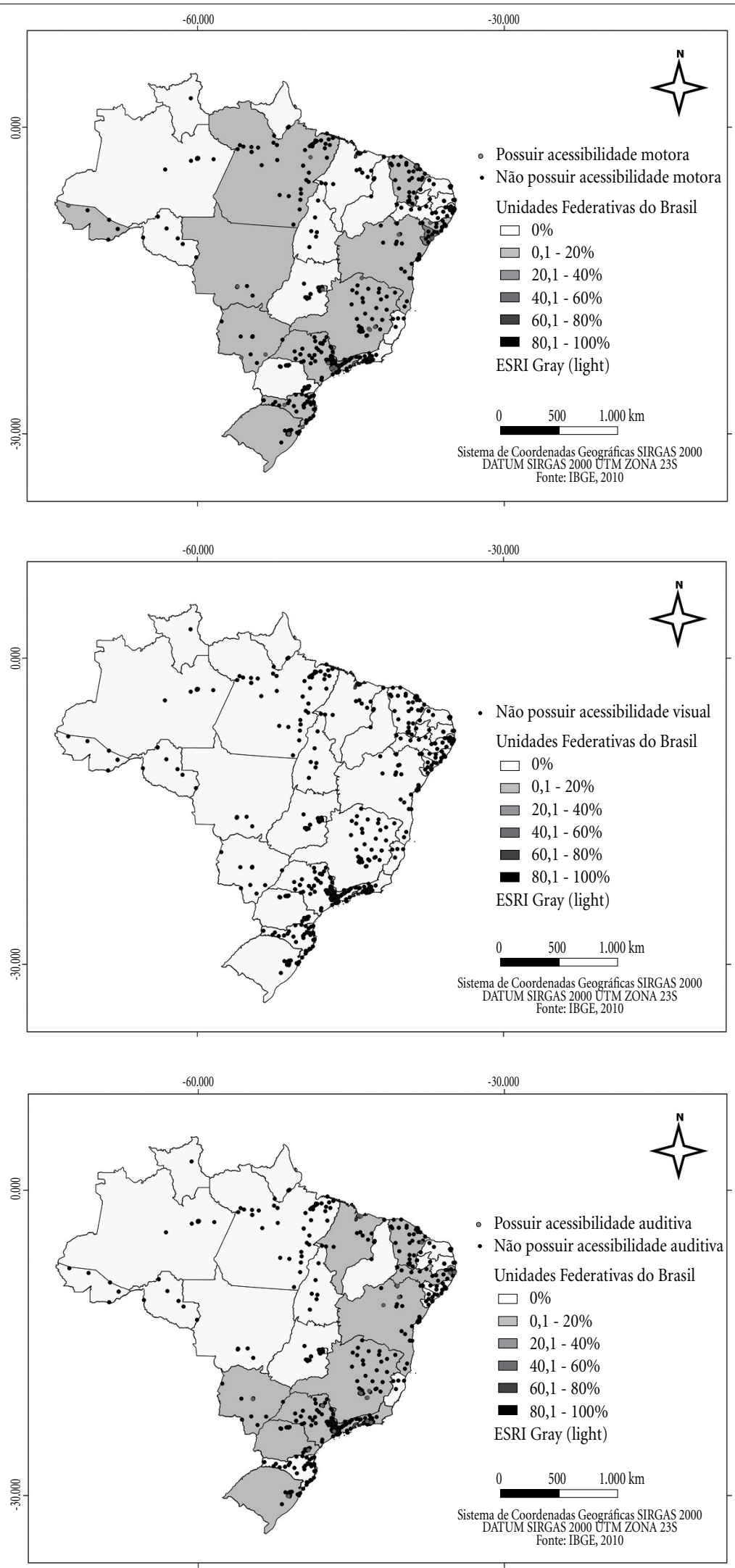

Figura 1. Acessibilidade a pessoas com deficiência motora (A), visual (B) e auditiva (C). Brasil, 2017. 
Tabela 2. Acessibilidade a pessoas com deficiência visual das maternidades vinculadas à Rede Cegonha. Brasil, 2017.

\begin{tabular}{|c|c|c|c|c|c|}
\hline $\begin{array}{l}\text { Unidade } \\
\text { Federativa }\end{array}$ & $\begin{array}{l}\text { No de Ma- } \\
\text { ternidades } \\
\text { Avaliadas }\end{array}$ & $\begin{array}{l}\text { Recepção } \\
\text { n (\%) }\end{array}$ & $\begin{array}{c}\text { Sala de } \\
\text { Admissão } \\
\text { n (\%) }\end{array}$ & $\begin{array}{c}\text { Unidade } \\
\text { Obstétrica } \\
\text { n (\%) }\end{array}$ & $\begin{array}{c}\text { Indicador de } \\
\text { Acessibilidade } \\
\text { Visual } \\
\text { n (\%) }\end{array}$ \\
\hline Norte & 86 & - & - & - & - \\
\hline Rondônia & 7 & - & - & - & - \\
\hline Acre & 6 & - & - & - & - \\
\hline Amazonas & 11 & - & - & - & - \\
\hline Roraima & 1 & - & - & - & - \\
\hline Pará & 47 & - & - & - & - \\
\hline Amapá & 7 & - & - & - & - \\
\hline Tocantins & 7 & - & - & - & - \\
\hline Nordeste & 175 & $3(1,7)$ & - & $3(1,7)$ & - \\
\hline Maranhão & 14 & $1(7,1)$ & - & $1(7,1)$ & - \\
\hline Piauí & 8 & $1(12,5)$ & - & - & - \\
\hline Ceará & 44 & - & - & - & - \\
\hline Rio Grande do Norte & 11 & - & - & - & - \\
\hline Paraíba & 10 & - & - & - & - \\
\hline Pernambuco & 32 & $1(3,1)$ & - & $1(3,1)$ & - \\
\hline Alagoas & 16 & - & - & $1(6,3)$ & - \\
\hline Sergipe & 9 & - & - & - & - \\
\hline Bahia & 31 & - & - & - & - \\
\hline Sudeste & 223 & $4(1,8)$ & $2(0,9)$ & $3(1,4)$ & - \\
\hline Minas Gerais & 44 & $1(2,3)$ & $1(2,3)$ & $1(2,3)$ & - \\
\hline Espírito Santo & 4 & - & - & - & - \\
\hline Rio de Janeiro & 41 & - & $1(2,4)$ & $1(2,4)$ & - \\
\hline São Paulo & 134 & $3(2,2)$ & - & $1(0,8)$ & - \\
\hline Sul & 81 & $1(1,2)$ & - & - & - \\
\hline Paraná & 23 & - & - & - & - \\
\hline Santa Catarina & 34 & $1(2,9)$ & - & - & - \\
\hline Rio Grande do Sul & 24 & - & - & - & - \\
\hline Centro-Oeste & 41 & $1(2,4)$ & - & - & - \\
\hline Mato Grosso do Sul & 11 & $1(9,1)$ & - & - & - \\
\hline Mato Grosso & 6 & - & - & - & - \\
\hline Goiás & 12 & - & - & - & - \\
\hline Distrito Federal & 12 & - & - & - & - \\
\hline Brasil & 606 & $9(1,5)$ & $2(0,3)$ & $6(1,0)$ & - \\
\hline
\end{tabular}

$30 \mathrm{~km}$ e $50 \mathrm{~km}$. Na distância de $100 \mathrm{~km}$, o estabelecimento localizado na Bahia, alcançou populações residentes do sul do estado do Piauí, do leste dos estados de Pernambuco, Alagoas e Sergipe, enquanto no outro estabelecimento de Minas Gerais, essa mesma distância (100km), alcançou a maior parte dos estados do Espírito Santo e Rio de Janeiro (Figura 2).

\section{Discussão}

O presente estudo aponta que a estrutura dos estabelecimentos hospitalares vinculados à $\mathrm{RC}$ no Brasil não está adaptada para pessoas com DM, DV ou DA, corroborando os achados de outros estudos nos quais foram observadas inadequações na estrutura física das unidades de saúde da família ${ }^{22,23}$.

A Política Nacional de Saúde da Pessoa com Deficiência $^{20}$, a Rede de Cuidados à Pessoa com 
Tabela 3. Acessibilidade a pessoas com deficiência auditiva das maternidades vinculadas à Rede Cegonha. Brasil, 2017.

\begin{tabular}{|c|c|c|c|c|c|}
\hline Unidade Federativa & $\begin{array}{l}\text { No de Ma- } \\
\text { ternidades } \\
\text { Avaliadas }\end{array}$ & $\begin{array}{c}\text { Recepção } \\
\text { n (\%) }\end{array}$ & $\begin{array}{c}\text { Sala de } \\
\text { Admissão } \\
\text { n (\%) }\end{array}$ & $\begin{array}{c}\text { Unidade } \\
\text { Obstétrica } \\
\text { n (\%) }\end{array}$ & $\begin{array}{c}\text { Indicador de } \\
\text { Acessibilidade } \\
\text { Auditiva } \\
\text { n (\%) }\end{array}$ \\
\hline Norte & 86 & $2(2,3)$ & - & $2(2,3)$ & - \\
\hline Rondônia & 7 & - & - & $1(14,3)$ & - \\
\hline Acre & 6 & - & - & $1(16,7)$ & - \\
\hline Amazonas & 11 & $2(18,2)$ & - & - & - \\
\hline Roraima & 1 & - & - & - & - \\
\hline Pará & 47 & - & - & - & - \\
\hline Amapá & 7 & - & - & - & - \\
\hline Tocantins & 7 & - & - & - & - \\
\hline Nordeste & 175 & $18(10,3)$ & $11(6,3)$ & $18(10,3)$ & $8(4,6)$ \\
\hline Maranhão & 14 & - & $3(21,4)$ & $3(21,4)$ & $2(14,3)$ \\
\hline Piauí & 8 & $1(12,5)$ & - & $1(12,5)$ & - \\
\hline Ceará & 44 & - & $2(4,6)$ & $7(15,9)$ & $1(2,3)$ \\
\hline Rio Grande do Norte & 11 & - & - & - & - \\
\hline Paraíba & 10 & - & - & - & - \\
\hline Pernambuco & 32 & $8(25,0)$ & $3(9,4)$ & $3(9,4)$ & $3(9,4)$ \\
\hline Alagoas & 16 & $2(12,5)$ & - & - & - \\
\hline Sergipe & 9 & - & - & - & - \\
\hline Bahia & 31 & $3(9,7)$ & $3(9,7)$ & $4(12,9)$ & $2(6,5)$ \\
\hline Sudeste & 223 & $26(11,7)$ & $18(8,1)$ & $44(19,7)$ & $9(4,0)$ \\
\hline Minas Gerais & 44 & $10(22,7)$ & $9(20,5)$ & $14(31,8)$ & $5(11,4)$ \\
\hline Espírito Santo & 4 & - & - & - & - \\
\hline Rio de Janeiro & 41 & $2(4,9)$ & $3(7,3)$ & $2(4,9)$ & $2(4,9)$ \\
\hline São Paulo & 134 & $14(10,5)$ & $6(4,5)$ & $28(20,9)$ & $2(1,5)$ \\
\hline Sul & 81 & $8(9,9)$ & $4(4,9)$ & $10(12,4)$ & $2(2,5)$ \\
\hline Paraná & 23 & $2(8,7)$ & $2(8,7)$ & $1(4,4)$ & $1(4,4)$ \\
\hline Santa Catarina & 34 & $2(5,9)$ & - & $2(5,9)$ & - \\
\hline Rio Grande do Sul & 24 & $4(16,7)$ & $2(8,3)$ & $7(29,2)$ & $1(4,2)$ \\
\hline Centro-Oeste & 41 & $5(12,2)$ & $3(7,3)$ & $11(26,8)$ & $1(2,4)$ \\
\hline Mato Grosso do Sul & 11 & $2(18,2)$ & $2(18,2)$ & $5(45,5)$ & $1(9,1)$ \\
\hline Mato Grosso & 6 & - & - & $4(66,7)$ & - \\
\hline Goiás & 12 & $1(8,3)$ & $1(8,3)$ & $1(8,3)$ & - \\
\hline Distrito Federal & 12 & $2(16,7)$ & - & $1(8,3)$ & - \\
\hline Brasil & 606 & $59(9,7)$ & $36(5,9)$ & $85(14,0)$ & $20(3,3)$ \\
\hline
\end{tabular}

O símbolo (-) representa valores iguais a zero. $\mathrm{n}(\%)=$ frequência absoluta e percentual.

Indicador de Acessibilidade Auditiva: reflete a proporção de estabelecimentos que estavam adequados nos três ambientes analisados (Recepção, Sala de Admissão, Unidade Obstétrica).

Deficiência $^{21}$ e a Lei Brasileira de Inclusão da Pessoa com Deficiência ${ }^{26}$ são marcos legais mais recentes destinados a assegurar e promover condições de igualdade, o exercício dos direitos sociais da pessoa com deficiência, visando à sua inclusão social e cidadania. Os resultados deste trabalho são um contraponto com esses marcos legais que enfatizam a importância do acesso e da qualidade dos serviços a esses indivíduos, equidade, integralidade do cuidado, aprimoramento da estrutura física dos estabelecimentos de saúde ${ }^{7}$, a disseminação de informações e uma visão dinâmica e global do estado de saúde de mulheres com DM, DV ou $\mathrm{DA}^{7,24}$.

É possível que problemas de gestão e financiamento do SUS dificultem a implementação mais efetiva de políticas públicas de saúde direcionadas às pessoas com deficiência, contribuindo para a baixa proporção de adequação dos estabelecimentos da Rede Cegonha para pessoas com deficiência ${ }^{36}$.

A qualificação dos serviços de saúde materno e infantil, especialmente para a população de 


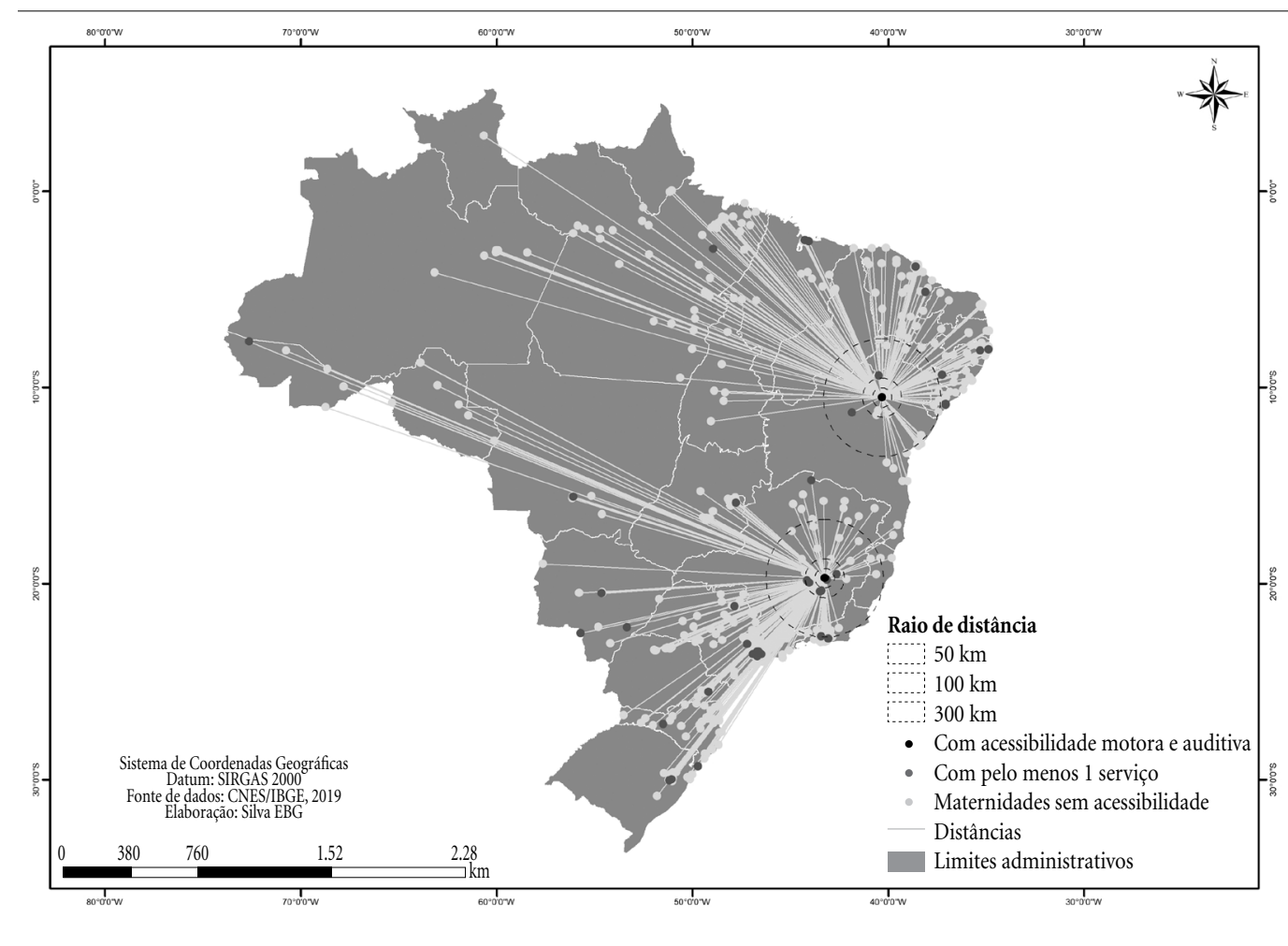

Figura 2. Distância dos hospitais com acessibilidade a pessoas com deficiência motora, visual e auditiva. Brasil, 2017.

maior vulnerabilidade, é uma estratégia importante para a redução das iniquidades em saúde e para o alcance de metas pactuadas internacionalmente. No Brasil, nas últimas décadas, essa temática vem recebendo maior destaque, sobretudo dado à persistência da elevada RMM, com estimativas acima dos padrões exigidos pela OMS, sendo o único Objetivo de Desenvolvimento do Milênio (ODM) que não foi alcançado pelo país ano de 2015 , não atingindo a redução em $70 \%$ no período de 1990 a $2015^{33}$.

Mulheres com necessidade especiais têm maior risco de apresentar problemas relacionacionados à gestação, parto e pós-parto em comparação com a população geral, como: taxas significativamente mais altas de depressão ${ }^{10}$, diabetes e infecções do trato urinário ${ }^{37}$ e de parto prematuro ${ }^{9,13,37}$ e baixo peso ao nascer ${ }^{9,13,37}$ entre os recém-nascidos. Portanto, há necessidade de melhorar a estrutura física das maternidades vinculadas à RC, para tornar $o$ acesso mais equitativo e otimizar os indicadores de saúde entre mulheres com DM, DV ou DA.

O Ministério da Saúde afirma ainda que as desigualdades regionais e entre grupos populacionais levam a diferentes RMM e que se faz necessário a construção e mensuração de estimativas confiáveis estratificadas por regiões e Unidades Federativas - UF, com o intuito de subsidiar o monitoramento de área prioritárias relacionadas à saúde da mulher, em especial gestantes, garantindo a implementação efetiva e avaliação das políticas públicas voltadas para essa população ${ }^{38}$.

Há mulheres com deficiência em todas as faixas etárias, raças, religiões, extratos econômicos e orientação sexual. Diz respeito, portanto, a um segmento da população que sofre com ações pouco expressivas voltadas para suas necessidades nos serviços de atenção à saúde. Elas são caracterizadas por possuírem uma dupla condição de vulnerabilidade, sob a perspectiva de que ser mulher e ter deficiência representam duas desvantagens sociais ${ }^{7}$.

Este estudo destaca que as maternidades das regiões Norte e Nordeste vinculadas à RC apresentaram maiores barreiras arquitetônicas para o acesso de gestantes com DM, DV ou DA. As desigualdades são maiores para a acessibilidade motora. Esse resultado sugere um reflexo das disparidades regionais em relação ao acesso aos serviços de saúde, em um contexto de baixo suporte destinado à atenção das gestantes/puérperas com deficiências físicas. Além disso, nessas regiões se 
concentram os piores indicadores socioeconômicos e de saúde, refletindo a dificuldade de se atingir a equidade no SUS para populações consideradas mais vulneráveis, sustentando a ideia de outros estudos que reiteram o pensamento de que as mulheres com DM, DV ou DA estão mais expostas à pobreza, desemprego e baixa escolaridade $2,7,8,38,39$.

As regiões Norte e Nordeste apresentam, ainda, uma maior proporção da população que tem acesso exclusivamente aos estabelecimentos de saúde do SUS, e com grandes barreiras de acesso a estabelecimentos de alta complexidade ${ }^{40}$, quase sempre com acesso restrito a hospitais mistos de menor complexidade ${ }^{41}$, com potenciais reflexos na mortalidade materna e neonatal. Além disso, a regionalização da assistência hospitalar ainda é considerada um desafio, dificultando a referência a locais com maior adequação da acessibilidade às mulheres com deficiências ${ }^{7,25,38}$.

Os estabelecimentos hospitalares das regiões Sul, Sudeste e Centro-Oeste apresentaram, em geral, melhores resultados, todavia, não atingindo proporções mínimas em vários dos critérios estruturais estudados. Esses resultados são indicativos de que uma parcela importante de gestantes/puérperas foi exposta a riscos evitáveis e desnecessários. Estudos reiteram que o padrão diferenciado das regiões supracitadas pode justificar-se pela excessiva concentração de maternidades nas capitais dos estados e a diferença de financiamento $6^{6,724,39}$.

Nenhum estabelecimento conseguiu apresentar todos os itens de estrutura adequados para gestantes/puérperas com DM, DV ou DA no Brasil. Os dois estabelecimentos que garantiram as acessibilidades motora e auditiva, alcançam populações residentes a uma distância menor $(30 \mathrm{~km})$ e intermediária $(50 \mathrm{~km})$, consideradas no presente estudo. Essas distâncias alcançam populações residentes dos estados da Bahia e Minas Gerais, em que se localizam tais estabelecimentos. Esse achado corrobora as desigualdades regionais encontradas, principalmente para a região Norte, cujas distâncias são enormes até o acesso a estabelecimentos com melhores condições de estrutura.

Apesar dos grandes avanços decorridos da implantação da RC no Brasil, especialmente nas boas práticas de atenção ao parto, poucas maternidades vinculadas à $\mathrm{RC}$ apresentaram adequação para gestantes e puérperas com DM, DV ou DA. Esse resultado é antagônico ao que prega a referida política, uma vez que esta encontra-se alicerçada nos componentes relacionados ao di- reito à gravidez, parto e puerpério seguros e humanizados e o direito ao planejamento reprodutivo, transporte e acessibilidade ${ }^{30,31}$.

Como limitação do presente estudo, apresenta-se a impossibilidade de inferências para o nível individual, haja vista o desenho ecológico do estudo, sob risco de falácia ecológica. Outra limitação refere-se ao fato de que o ambiente alojamento conjunto não foi estudado para a caracterização da acessibilidade visual e auditiva, mas, somente para a acessibilidade motora. Apesar disso, a proporção de estabelecimentos com acessibilidade motora (adequado para todos os critérios analisados em todos os ambientes estudados) foi maior do que com acessibilidade visual e auditiva. Os critérios empregados para caracterizar acessibilidade não incluíram todas as normas preconizadas na NBR-9050 $0^{42}$. Ainda assim, mesmo considerando somente o mínimo possível, os resultados foram muito insatisfatórios. Cabe destacar ainda que os padrões elegidos ao longo deste trabalho são aqueles que refletem os parametros de acessibilidades mínimos. A utilização de diretrizes mais completas revelaria um cenário ainda mais desafiador, do ponto de vista de acessibilidade.

Este trabalho apresenta pontos fortes como: a criação de mapas para apresentar o panorama da acessibilidade motora, visual e auditiva para gestantes e púerperas, o que permite identificar a distribuição geográfica dos estabelecimentos no território nacional, e uma melhor visualização das barreiras de acesso. Além disso, este estudo descreveu a acessibilidade em todos os estabelecimentos vinculados à RC, analisando sem precedentes na literatura a acessibilidade para gestantes e puérperas com DM, DV ou DA em estabelecimentos hospitalares/maternidades de todo o território brasileiro.

\section{Conclusão}

A estrutura dos estabelecimentos hospitalares vinculados à $\mathrm{RC}$ não está adaptada para pessoas com DM, DV ou DA no Brasil, especialmente nas regiões Norte e Nordeste. Apesar dos avanços normativos quanto à inclusão de pessoas com deficiência nas políticas públicas, ainda persistem desafios quanto à equidade no SUS, para essa população. Portanto, adequar a estrutura física desses estabelecimentos hospitalares às necessidades dessa população pode contribuir para melhorar indicadores de saúde materna e infantil. 


\section{Colaboradores}

EBAF Thomaz - Concepção do Estudo, Coleta dos Dados, Desenvolvimento da Metodologia, Análise dos Dados, Processo de Escrita do Artigo e Revisão Crítica do Texto Final. EM Costa Análise dos Dados, Processo de Escrita do Artigo e Revisão Crítica do Texto Final. YNLA Goiabeira - Processo de Escrita do Artigo e Revisão Crítica do Texto Final. TAH Rocha, NCS Rocha e RCS Queiroz - Desenvolvimento da Metodologia, Análise dos Dados e Revisão Crítica do Texto Final. COM Marques - Concepção do Estudo, Coleta dos Dados, Desenvolvimento da Metodologia e Revisão Crítica do Texto Final.

\section{Referências}

1. Instituto Brasileiro de Geografia e Estatística (IBGE). Censo Demográfico 2010: Características gerais da população, religião e pessoas com deficiência. [acessado 2019 Jun 6]. Disponível em: http://www.ibge.gov.br/ home/estatistica/populacao/censo2010/caracteristicas_religiao_deficiencia/caracteristicas_religiao_deficiencia_tab_gregioes_xls.shtm

2. Mathias K, Kermode M, San Sebastian M, Davar B, Goicolea I. An asymmetric burden: Experiences of men and women as caregivers of people with psychosocial disabilities in rural North India. Transcult Psychiatry 2019; 56(1):76-102.

3. Belzunegui-Eraso A, Pastor-Gosálbez I, Puig-Andreu X, Valls-Fonayet F. Risk of Exclusion in People with Disabilities in Spain: Determinants of Health and Poverty. Int J Environ Res Public Health 2018; 15(10):2129.

4. Mitra M, Akobirshoev I, Moring NS, Long-Bellil L, Smeltzer SC, Smith LD, Iezzoni LI. Access to and Satisfaction with Prenatal Care Among Pregnant Women with Physical Disabilities: Findings from a National Survey. J Womens Health (Larchmt) 2017; 26(12):1356-1363.

5. Boccolini PMM, Duarte CMR, Marcelino MA, Boccolini CS. Social inequalities in limitations caused by chronic diseases and disabilities in Brazil: the 2013 National Health Survey. Cien Saude Colet 2017; 22(11):3537-3546.

6. Gudlavalleti MVS, John N, Allagh K, Sagar J, Kamalakannan S, Ramachandra SS. Access to health care and employment status of people with disabilities in South India, the SIDE (South India Disability Evidence) study. BMC public health 2014; 14(1):1125.

7. Nicolau SM, Schraiber LB, Ayres JRDCM. Mulheres com deficiência e sua dupla vulnerabilidade: contribuições para a construção da integralidade em saúde. Cien Saude Colet 2013; 18(3):863-872.
8. Devkota HR, Kett M, Groce N. Societal attitude and behaviours towards women with disabilities in rural Nepal: pregnancy, childbirth and motherhood. $B M C$ Pregnancy Childbirth 2019; 19(1):20.

9. Long-Bellil L, Mitra M, Iezzoni LI, Smeltzer SC, Smith L. The Impact of Physical Disability on Pregnancy and Childbirth. J Womens Health (Larchmt) 2017; 26(8):878-885.

10. Murthy GVS, John N, Sagar J. Reproductive health of women with and without disabilities in South India, the SIDE study (South India Disability Evidence) study: a case control study. BMC Women's Health 2014; 14(1):146.

11. Tarasoff LA, Ravindran S, Malik H, Salaeva D, Brown HK. Maternal disability and risk for pregnancy, delivery, and postpartum complications: A systematic review and meta-analysis. Am J Obstet Gynecol 2019; 222(1):27.

12. Andresen EM, Peterson-Besse JJ, Krahn GL, Walsh ES, Horner-Johnson W, Iezzoni LI. Pap, mammography, and clinical breast examination screening among women with disabilities: A systematic review. Womens Health Issues 2013; 23(14):205-214.

13. Iezzoni LI, Kilbridge K, Park ER. Physical access barriers to care for diagnosis and treatment of breast cancer among women with mobility impairments. Oncol Nurs Forum 2010; 37(6):711-717.

14. McRee A-L, Haydon AA, Halpern CT. Reproductive health of young adults with physical disabilities in the U.S. Prev Med 2010; 51(6):502-504.

15. Drainoni ML, Lee-Hood E, Tobias C, Bachman SS, Andrew J, Maisels L. Cross-disability experiences of barriers to health-care access: Consumer perspectives. J Disabil Policy Stud 2006; 17(2):101-115.

16. Read S, Heslop P, Turner S, Mason-Angelow V, Tilbury N, Miles C, Hatton C. Disabled people's experiences of accessing reasonable adjustments in hospitals: a qualitative study. BMC Health Serv Res 2018; 18(1):931. 
17. Sakellariou D, Rotarou ES. The effects of neoliberal policies on access to healthcare for people with disabilities. Int J Equity Health 2017; 16(1):199.

18. Hunt X, Carew MT, Braathen SH, Swartz L, Chiwaula $\mathrm{M}$, Rohleder $\mathrm{P}$. The sexual and reproductive rights and benefit derived from sexual and reproductive health services of people with physical disabilities in South Africa: beliefs of non-disabled people. Reprod Health Matters 2017; 25(50):66-79.

19. Brasil. Portaria $n^{\circ} 1.060$, de 5 de junho de 2002. Institui a Política Nacional de Saúde da Pessoa Portadora de Deficiência. Diário Oficial da União 2002; 5 jun.

20. Brasil. Ministério da Saúde (MS). Política Nacional de Saúde da Pessoa com Deficiência. Brasília: Editora do Ministério da Saúde; 2010. [acessado 2020 Maio 26]. Disponível em: https://bvsms.saude.gov.br/bvs/ publicacoes/politica_nacional_pessoa_com_deficiencia.pdf

21. Brasil. Portaria no 793, de 24 de abril de 2012. Institui a Rede de Cuidados à Pessoa com Deficiência no âmbito do Sistema Único de Saúde. Diário Oficial da União 2012; 24 abr.

22. Martins KP, Costa TFD, Medeiros TMD, Fernandes MDGM, França ISXD, Costa K. NDFM. Estrutura interna de Unidades de Saúde da Família: acesso para as pessoas com deficiência. Cien Saude Colet 2016; 21(10):3153-3160.

23. Siqueira FCV, Facchini LA, Silveira DSD, Piccini RX, Thumé E, Tomasi E. Barreiras arquitetônicas a idosos e portadores de deficiência física: um estudo epidemiológico da estrutura física das unidades básicas de saúde em sete estados do Brasil. Cien Saude Colet 2009; 14(1):39-44.

24. Bittencourt SDA, Domingues RMSM, Reis LGC, Ramos MM, Leal CM. Adequacy of public maternal care services in Brazil. Reprod Health 2016; 13(3):120.

25. Bittencourt SDA, Reis LGC, Ramos MM, Rattner D, Rodrigues PL, Neves DCO, Leal MDC. Estrutura das maternidades: aspectos relevantes para a qualidade da atenção ao parto e nascimento. Cad Saude Publica 2014; 30(1):208-219.

26. Brasil. Lei $\mathrm{n}^{\circ} 13.146$, de 06 de julho de 2015. Institui a Lei Brasileira de Inclusão da Pessoa com Deficiência (Estatuto da Pessoa com Deficiência). Diário Oficial da União 2015; 7 jul.

27. Instituto Brasileiro de Geografia e Estatística (IBGE). Síntese dos indicadores sociais: uma análise das condições de vida da população brasileira. Rio de Janeiro: IBGE; 2016. [acessado 2019 Jun 28]. Disponível em: https://biblioteca.ibge.gov.br/visualizacao/livros/ liv98965.pdf.

28. Instituto Brasileiro de Geografia e Estatística (IBGE). Censo demográfico: características gerais da população, religião e pessoas com deficiência. Rio de Janeiro: IBGE; 2010. [acessado 2019 Jun 27]. Disponível em: https:// www.ibge.gov.br/estatisticas/economicas/contas-nacionais/9088-produto-interno-bruto-dos-municipios.html $=\& \mathrm{t}=$ series-historicas\& utm_source $=$ landing\&utm_medium $=$ explica\&utm_campaign $=$ pib

29. Brasil. Ministério da Saúde (MS). Saúde Brasil 2017: uma análise da situação de saúde e os desafios para o alcance dos objetivos de desenvolvimento sustentável. Brasília: MS; 2018. [acessado 2019 Jun 28]. Disponível em: http://bvsms.saude.gov.br/bvs/publicacoes/ saude_brasil_2017_analise_situacao_saude_desafios_ objetivos_desenvolvimento_sustetantavel.pdf
30. Brasil. Ministério da Saúde (MS). Humanização do parto e do nascimento. Universidade Estadual do Ceará. Brasília: 2014 [acessado 2018 Mar 29]. Disponível em: http://bvsms.saude.gov.br/bvs/publicacoes/parto. pdf

31. Brasil. Ministério da Saúde (MS). Manual prático para implementação da Rede Cegonha. Brasília: MS; 2011.

32. Organização Mundial de Saúde (OMS). Maternidade Segura. Assistência ao Parto Normal: um guia prático. Genebra: OMS; 1996.

33. Brasil. Ministério da Saúde (MS). Diretrizes de Atenção à Gestante: a operação cesariana 2015. [acessado 2019 Jul 8]. Disponível em: http://conitec.gov.br/ images/Relatorios/2016/Relatorio_Diretrizes-Cesariana_final.pdf.

34. Brasil. Ministério da Saúde (MS). Diretrizes nacionais de assistência ao parto normal: versão resumida. Brasília: MS; 2017 [acessado 2018 Mar 29]. Disponível em: http://bvsms.saude.gov.br/bvs/publicacoes/diretrizes_nacionais_assistencia_parto_normal.pdf .

35. Qgis Training Manual. [acessado 2019 Ago 21]. Disponível: https://docs.qgis.org/3.4/pdf/en/QGIS-3.4QGISTrainingManual-en.pdf

36. Paim J, Travassos C, Almeida C, Bahia L, Macinko J. The Brazilian health system: history, advances, and challenges. Lancet 2011; 377(9779):1778 1797.

37. Morton C, Le JT, Shahbandar L, Hammond C, Murphy EA, Kirschner KL. Pregnancy outcomes of women with physical disabilities: a matched cohort study. PMßR 2013; 5(2):90-98.

38. Brasil. Ministério da Saúde (MS). Saúde Brasil 2017: uma análise da situação de saúde e os desafios para o alcance dos objetivos de desenvolvimento sustentável. Brasília: MS; 2018.

39. Almeida WS, Szwarcwald CL. Mortalidade infantil e acesso geográfico ao parto dos municípios brasileiros. Rev Saude Publica 2012; 46(1):68-76.

40. Rocha TAH, Silva NC, Amaral PV, Barbosa ACQ, Rocha JVM, Alvares V, Almeida DG, Thumé E, Thomaz EBAF, Queiroz RCS, Souza MR, Lein A, Lopes DP, Staton C, Vissoci JRN, Facchini LA. Addressing geographic access barriers to emergency care services: a national ecologic study of hospitals in Brazil. Int $J$ Equit Health 2017; 16(1):149.

41. Rocha TAH, Silva NC, Amaral PV, Barbosa ACQ, Rocha JVM, Alvares V, Almeida DG, Thumé E, Tomaz EBAF, Queiroz RCS, Vissoci JRN, Souza MR, Lein A, Staton C, Facchini LA. Access to emergency care services: A transversal ecological study about Brazilian emergency health care network Public Health. Public Health 2017; 153:9-15.

42. Norma Brasileira Regulamentadora (NBR). Acessibilidade a edficações, mobiliário, espaços e equipamentos urbanos [recurso eletrônico]. Rio de Janeiro: 2015. [acessado 2017 Ago 7]. Disponível em: https://www. ufpb.br/cia/contents/manuais/abnt-nbr9050-edicao-2015.pdf

Artigo apresentado em 30/03/2020

Aprovado em 03/07/2020

Versão final apresentada em 05/07/2020

Editores-chefes: Romeu Gomes, Antônio Augusto Moura da Silva 